VOL. 57 (1998) [109-115]

\title{
SEMI-SYMMETRIC BALL-HOMOGENEOUS SPACES AND A VOLUME CONJECTURE
}

\author{
G. Calvaruso and L. Vanhecke
}

\begin{abstract}
We prove that semi-symmetric ball-homogeneous spaces are locally symmetric and we use this result to prove that a semi-symmetric Riemannian manifold such that the volume of each sufficiently small geodesic ball is the same as in a Euclidean space, is locally flat.
\end{abstract}

\section{INTRODUCTION}

A Riemannian manifold $(M, g)$ is said to be semi-symmetric if at each point of $M$ the Riemann curvature tensor is the same as that of a symmetric space, which may vary with the point. This class of manifolds extends that of the locally symmetric spaces. For a thorough treatment, historical remarks and further references, we refer to [3]. The main aim of this paper is to give for this class of manifolds a positive answer to two open problems which we describe now.

Riemannian manifolds $(M, g)$ equipped with a transitive pseudogroup of isometries are called locally homogeneous spaces. Clearly, such spaces have the property that the volumes of sufficiently small geodesic spheres or balls only depend on the radius. An $(M, g)$ for which this holds is called a ball-homogeneous space [11]. Up to now we do not know of any example of a ball-homogeneous space which is not locally homogeneous and this raises the first problem: Is a ball-homogeneous Riemannian manifold necessarily locally homogeneous or not? Positive answers are known for several special cases $[4,5,7$, 13], but the general problem remains open. Since semi-symmetric locally homogeneous spaces are locally symmetric [1], it is worthwhile to investigate whether this also holds for semi-symmetric ball-homogeneous spaces. We shall provide a positive answer obtaining in this way that any semi-symmetric ball-homogeneous space is indeed locally homogeneous.

Using this result, we are then able to provide for the class of semi-symmetric spaces a positive answer to the following problem stated in [8]: Let $(M, g)$ be a Riemannian manifold such that the volume of each geodesic ball of sufficiently small radius is the same as in Euclidean space. Is $(M, g)$ locally flat? Also here we have only partial

Received 23rd April, 1997

First author supported by grant decr. n. 1106 of 26/09/1995 of Università degli Studi di Lecce (Italy).

Copyright Clearance Centre, Inc. Serial-fee code: 0004-9729/98 \$A2.00+0.00. 
answers and the general case is still an intriguing open problem. Our result extends that of $[6,8]$ for the class of locally symmetric spaces. It should be noted that an $(M, g)$ satisfying the hypothesis in the above problem is necessarily ball-homogeneous.

In Section 2 we start by collecting some fundamental results concerning the local structure of semi-symmetric spaces. Next, we consider the ball-homogeneity for this class in Section 3 and finally, we treat the volume conjecture in Section 4.

\section{SEMI-SYMMETRIC SPACES}

Let $(M, g)$ be a smooth, connected Riemannian manifold with Levi Civita connection $\nabla$ and Riemannian curvature tensor $R$ given by $R_{X Y}=\nabla_{[X, Y]}-\left[\nabla_{X}, \nabla_{Y}\right]$ for all smooth vector fields $X, Y$. We denote by $\varrho$ and $\tau$, respectively the Ricci tensor of type $(0,2)$ and the scalar curvature of $(M, g)$.

$(M, g)$ is said to be semi-symmetric if $R$ satisfies $R_{X Y} \cdot R=0$ for all vector fields $X$, $Y$ and where $R_{X Y}$ acts as a derivation on $R$. As already mentioned in the Introduction, this is equivalent to saying that $R_{p}$ is, for each $p \in M$, the same as the curvature tensor of a symmetric space. This last space may vary with $p$. The following proposition describes the local structure of $(M, g)$.

PRoposition 2.1. [12] There exists an open dense subset $U$ of $M$ such that around every point of $U$ the manifold is locally isometric to the direct product of symmetric spaces, two-dimensional manifolds, spaces foliated by Euclidean leaves of codimension two, elliptic cones, hyperbolic cones, Euclidean cones and Kählerian cones.

Further, we have (since the spaces of "cone type" do not have constant scalar curvature)

PROPOSITION 2.2. [10] If $\tau$ is constant, then on $U$ the semi-symmetric space $(M, g)$ is locally isometric to a Riemannian product

$$
\left(M_{s}, g_{s}\right) \times\left(M_{1}, g_{1}\right) \times \ldots \times\left(M_{r}, g_{r}\right)
$$

where $\left(M_{s}, g_{s}\right)$ is a symmetric space and $\left(M_{i}, g_{i}\right), i=1, \ldots, r$, are locally irreducible Riemannian spaces foliated by totally geodesic Euclidean leaves of codimension two and have constant scalar curvature.

Note that the semi-symmetric space $(M, g)$ has constant scalar curvature on $U$ if and only if $(M, g)$ is curvature homogeneous on $U$, that is, for each pair of points $p, q \in U$, there exists a linear isometry $\varphi: T_{p} U \rightarrow T_{q} U$ such that $\varphi^{*} R_{q}=R_{p}$.

For more details and references we refer to [3] where the following result concerning the explicit description of the metric of an $\left(M_{i}, g_{i}\right)$ is given.

PRoposition 2.3. [2, 3] Let $(M, g)$ be an $(n+2)$-dimensional locally irreducible semi-symmetric space foliated by n-dimensional Euclidean leaves and such that 
its scalar curvature is constant along each leaf. Then there exists a dense open subset $U$ of $F$ such that in a neighbourhood of every point $p \in U$ there exist local coordinates $\left(w, x^{1}, \ldots, x^{n+1}\right)$ and an orthonormal coframe of the form

$$
\left\{\begin{aligned}
\omega^{0} & =f\left(w, x^{1}\right) d w \\
\omega^{i} & =d x^{i}+\sum_{j=1}^{n+1} D_{j}^{i}(w) x^{j} d w, \quad i=1, \ldots, n+1,
\end{aligned}\right.
$$

where $D_{j}^{i}(w)+D_{i}^{j}(w)=0$. The scalar curvature of this metric is given by

$$
\tau=-2 f^{-1} f_{x^{1} x^{1}}^{\prime \prime} \neq 0
$$

Conversely, any local metric of this form is semi-symmetric and foliated by Euclidean leaves of codimension two with constant scalar curvature along the leaves.

\section{BALL-HOMOGENEOUS SEMI-SYMMETRIC SPACES}

Let $(M, g)$ be a Riemannian manifold and $m \in M$. Further, let $B_{m}(r)$ denote a geodesic ball centred at $m$ with sufficiently small radius $r$. We denote by $V_{m}(r)$ the volume of $B_{m}(r)$. Then $(M, g)$ is said to be ball-homogeneous if $V_{m}(r)$ is independent of $m$. As already mentioned, locally homogeneous spaces are trivial examples and it is still an open problem whether there are ball-homogeneous spaces which are not locally homogeneous. In what follows we shall prove that this is not the case inside the class of semi-symmetric spaces.

To prepare for the proof we first note that the condition of ball-homogeneity implies conditions on an infinite number of scalar curvature invariants. Indeed, the condition implies that all the derivatives $\frac{d^{k} V_{m}(r)}{d r}, k \in \mathbb{N}$, evalueted at $m$, have to be globally constant and these derivatives are expressions of scalar curvature invariants. For arbitrary $k$ these explicit expressions are not known. Nevertheless, we have [8] for any $n$-dimensional manifold:

$$
V_{m}(r)=\omega r^{n}\left(1+A(m) r^{2}+B(m) r^{4}+C(m) r^{6}+O\left(r^{8}\right)\right)
$$

where

$$
\begin{aligned}
A(m)= & -\frac{\tau(m)}{6(n+2)}, \\
B(m)= & \frac{\left(-3\|R\|^{2}+8\|\varrho\|^{2}+5 \tau^{2}-18 \tau \Delta \tau\right)(m)}{360(n+2)(n+4)}, \\
C(m)= & \frac{1}{720(n+2)(n+4)(n+6)}\left\{-\frac{5}{9} \tau^{3}-\frac{8}{3} \tau\|\varrho\|^{2}\right. \\
& +\tau\|R\|^{2}+\frac{64}{63} \check{\varrho}-\frac{64}{21} g(\varrho \otimes \varrho, \bar{R})+\frac{32}{7} g(\varrho, \dot{R})
\end{aligned}
$$




$$
\begin{aligned}
& -\frac{110}{63} \check{R}-\frac{200}{63} \check{\bar{R}}+\frac{45}{7}\|\nabla \tau\|^{2}+\frac{45}{14}\|\nabla \varrho\|^{2} \\
& +\frac{45}{7} \alpha(\varrho)-\frac{45}{14}\|\nabla R\|^{2}+6 \tau \Delta \tau+\frac{48}{7} g(\Delta \varrho, \varrho) \\
& \left.+\frac{54}{7} g\left(\nabla^{2} \tau, \varrho\right)-\frac{30}{7} g(\Delta R, R)-\frac{45}{7} \Delta^{2} \tau\right\}(m)
\end{aligned}
$$

We also refer to [8] for the definitions of the invariants appearing in these expressions. Note that $\omega$ is the volume of a ball of radius 1 in Euclidean space.

This implies

LEMMA 3.1. Let $(M, g)$ be a semi-symmetric ball-homogeneous space. Then the invariants $\tau$ and $5\|\nabla R\|^{2}-17\|\nabla \varrho\|^{2}+30 \alpha(\varrho)$ are globally constant. Moreover, $(M, g)$ is curvature homogeneous on $U$.

Proof: Since $(M, g)$ is ball-homogeneous and connected, $A$, and hence $\tau$, must be constant on $M$. So, $(M, g)$ is curvature homogeneous on $U$. This implies that all scalar curvature invariants which do not involve the components of $\nabla R$ are globally constant on $M$. The result follows then at once from the constancy of $C$ and the relations

$$
g(\Delta \varrho, \varrho)=\frac{1}{2} \Delta\|\varrho\|^{2}-\|\nabla \varrho\|^{2}, \quad g(\Delta R, R)=\frac{1}{2} \Delta\|R\|^{2}-\|\nabla R\|^{2} .
$$

Using this, we are now in a position to prove the main result of this section.

TheOREM 3.2. A semi-symmetric ball-homogeneous space is locally symmetric.

Proof: It follows from Lemma 3.1 that $\tau$ is constant on $M$. Hence, the manifold may be described locally as in Proposition 2.2. Since $\tau$ and $5\|\nabla R\|^{2}-17\|\nabla \varrho\|^{2}+30 \alpha(\varrho)$ are additive curvature invariants, it follows that the corresponding invariants for each factor $M_{i}$ are also constant on the factor. In the following lemma we shall prove that this implies that each of the factors is locally symmetric. Since $M$ is connected, it then follows from Proposition 2.2 that $(M, g)$ is locally symmetric.

So, we are left with

LEMma 3.3. Let $(M, g)$ be a locally irreducible curvature homogeneous semisymmetric space foliated by totally geodesic Euclidean leaves of codimension two and such that the invariant $5\|\nabla R\|^{2}-17\|\nabla \varrho\|^{2}+30 \alpha(\varrho)$ is constant on $M$. Then $(M, g)$ is locally symmetric.

Proof: We use the local coordinates and the orthonormal coframe given in Proposition 2.3. Using the standard formulas (see for example [9]) we easily obtain the connection forms $\omega_{i}^{j}$ :

$$
\begin{cases}\omega_{1}^{0}=f^{-1} f_{x_{1}}^{\prime} \omega^{0}, & \\ \omega_{j}^{0}=0, & j \geqslant 2, \\ \omega_{j}^{i}=f^{-1} D_{j}^{i}(w) \omega^{0}, & i, j \geqslant 1,\end{cases}
$$


where $\omega_{j}^{i}+\omega_{i}^{j}=0$. Then the Riemann curvature tensor and the Ricci tensor of $(M, g)$ are given by

$$
\begin{gathered}
R=2 \tau\left(\omega^{0} \wedge \omega^{1} \otimes \omega^{0} \wedge \omega^{1}\right), \\
\varrho=\frac{\tau}{2}\left(\omega^{0} \otimes \omega^{0}+\omega^{1} \otimes \omega^{1}\right)
\end{gathered}
$$

(see also [3]).

Next, let $\left\{e_{0}, \ldots, e_{n+1}\right\}$ be the local orthonormal frame dual to $\left\{\omega^{0}, \ldots, \omega^{n+1}\right\}$ and let $X$ be a smooth vector field on $M$. Since

$$
\nabla_{X} \omega^{i}=-\sum_{j=0}^{n+1} \omega_{j}^{i}(X) \omega^{j}, \quad i=0, \ldots, n+1
$$

we obtain

$$
\left\{\begin{array}{l}
\nabla_{X} \omega^{0}=-\omega^{0}(X) f^{-1} f_{x_{1}}^{\prime} \omega^{1} \\
\nabla_{X} \omega^{1}=\omega^{0}(X) f^{-1}\left[f_{x_{1}}^{\prime} \omega^{0}-\sum_{j=2}^{n+1} D_{j}^{1} \omega^{j}\right]
\end{array}\right.
$$

From this and the expression of $\nabla R$ we get

$$
\nabla_{X} R=-2 \tau \omega^{0}(X) f^{-1} \sum_{j=2}^{n+1} D_{j}^{1}(w)\left(\omega^{0} \wedge \omega^{j} \otimes \omega^{0} \wedge \omega^{1}+\omega^{0} \wedge \omega^{1} \otimes \omega^{0} \wedge \omega^{j}\right) .
$$

In particular, this yields

$$
\left\{\begin{array}{l}
\nabla_{e_{0}} R=-2 \tau f^{-1} \sum_{j=2}^{n+1} D_{j}^{1}(w)\left(\omega^{0} \wedge \omega^{j} \otimes \omega^{0} \wedge \omega^{1}+\omega^{0} \wedge \omega^{1} \otimes \omega^{0} \wedge \omega^{j}\right) \\
\nabla_{e_{k}} R=0, \quad k \geqslant 1 .
\end{array}\right.
$$

So, the only possible non-vanishing components $\nabla_{r} R_{i j k l}=\nabla_{e_{r}} R\left(e_{i}, e_{j}, e_{k}, e_{l}\right)$ of $\nabla R$ are

$$
\nabla_{0} R_{10 j 0}=-\frac{1}{2} \tau f^{-1} D_{j}^{1} \quad \text { for } j \geqslant 2,
$$

and those obtained from this one by using the symmetry properties of $\nabla R$. This yields

$$
\|\nabla R\|^{2}=2 \tau^{2}\left(f^{-1}\right)^{2} \sum_{j=2}^{n+1}\left(D_{j}^{1}\right)^{2} .
$$

From the expression of $\nabla R$ we then derive for $\nabla \varrho$ :

$$
\begin{cases}\nabla_{0} \varrho_{1 j}=\nabla_{0} \varrho_{j 1}=-\frac{\tau}{2} f^{-1} D_{j}^{1} & \text { for } j \geqslant 2, \\ \nabla_{i} \varrho_{j k}=0 & \text { in all the other cases . }\end{cases}
$$


Hence, we get

$$
\begin{aligned}
& \|\nabla \varrho\|^{2}=\frac{\tau^{2}}{2}\left(f^{-1}\right)^{2} \sum_{j=2}^{n+1}\left(D_{j}^{1}\right)^{2}, \\
& \alpha(\varrho)=\sum_{i, j, k=0}^{n+1} \nabla_{i} \varrho_{j k} \nabla_{j} \varrho_{i k} \doteq 0 .
\end{aligned}
$$

So, we finally get

$$
5\|\nabla R\|^{2}-17\|\nabla \varrho\|^{2}+30 \alpha(\varrho)=\frac{3}{2} \tau^{2}\left(f^{-1}\right)^{2} \sum_{j=2}^{n+1}\left(D_{j}^{1}\right)^{2} .
$$

Since $D_{j}^{1}$ depends only on $w$ and $f$ depends on $x^{1}$, the constancy of this invariant implies that $D_{j}^{1}=0$ for all $j=2, \ldots, n+1$. So, $\|\nabla R\|^{2}=0$, which implies the required result. $\square$

\section{SEMI-SYMMETRIC SPACES AND THE VOLUME CONJECTURE}

Now we turn to the conjecture " $V_{m}(r)=\omega r^{n} \Rightarrow$ local flatness" stated in [8]. Up to now we only know partial answers and the general conjecture seems to be a very difficult one. Nevertheless, it is proved in $[6,8]$ that the conjecture holds for locally symmetric spaces. It now follows at once from this result and Theorem 3.2 that the conjecture is also true for the broader class of semi-symmetric spaces, since an $(M, g)$ which satisfies the hypothesis of the conjecture is necessarily a ball-homogeneous space. Hence, we have

THEOREM 4.1. Let $(M, g)$ be a semi-symmetric space such that the volume of all sufficiently small geodesic spheres or balls is the same as in Euclidean space. Then $(M, g)$ is locally flat.

\section{REFERENCES}

[1] E. Boeckx, 'Einstein-like semi-symmetric spaces', Arch. Math. (Brno) 29 (1993), 235-240.

[2] E. Boeckx, O. Kowalski and L. Vanhecke, 'Non-homogeneous relatives of symmetric spaces', Differential Geom. Appl. 4 (1994), 45-69.

[3] E. Boeckx, O. Kowalski and L. Vanhecke, Riemannian manifolds of conullity two (World Scientific Publ. Co., Singapore, 1996).

[4] G. Calvaruso, Ph. Tondeur and L. Vanhecke, 'Four-dimensional ball-homogeneous and C-spaces', Beiträge Algebra Geom. (to appear).

[5] G. Calvaruso and L. Vanhecke, 'Special ball-homogeneous spaces', Z. Anal. Anwendungen (to appear).

[6] M. Ferrarotti and L. Vanhecke, 'Locally symmetric spaces and the volume conjecture for geodesic balls', Math. J. Toyama Univ. 14 (1991), 89-93.

[7] E. Garcia-Río and L. Vanhecke, 'Five-dimensional $\varphi$-symmetric spaces', Balkan J. Geom. Appl. (to appear). 
[8] A. Gray and L. Vanhecke, 'Riemannian geometry as determined by the volumes of small geodesic balls', Acta Math. 142 (1979), 157-198.

[9] S. Kobayashi and K. Nomizu, Foundations of differential geometry I, II (Interscience Publ., New York, 1963, 1969).

[10] O. Kowalski, F. Tricerri and L. Vanhecke, 'Curvature homogeneous Riemannian manifolds', J. Math. Pure Appl. 71 (1992), 471-501.

[11] O. Kowalski and L. Vanhecke, 'Ball-homogeneous and disk-homogeneous Riemannian manifolds', Math. Z. 180 (1982), 429-444.

[12] Z. I. Szabó, 'Structure theorems on Riemannian manifolds satisfying $R(X, Y) \cdot R=0$, I, the local version', J. Differential Geom. 17 (1982), 531-582.

[13] L. Vanhecke, 'Scalar curvature invariants and local homogeneity', in Proc. Workshop on Differential Geometry and Topology (dedicated to the memory of Franco Tricerri) Palermo 1996 (to appear).

Department of Mathematics

Katholieke Universiteit Leuven

Celestijnenlaas $200 \mathrm{~B}$

3001 Leuven

Belgium 